\title{
Gemeinsame Stellungnahme der Deutschen Gesellschaft für Pneumologie und Beatmungsmedizin und der Deutschen Röntgengesellschaft zur Lungen- krebsfrüherkennung mit Niedrigdosis-CT
}

\author{
Joint Statement of the German Respiratory Society and the German Roentgenological Society on the \\ Early Detection of Lung Cancer by Low-Dose CT
}

Autoren

\author{
C. Vogelmeier ${ }^{1}$, H. Worth ${ }^{1}$, M. Pfeifer ${ }^{1}$, O. Karg ${ }^{1}$ (Vorstand), M. Reck ${ }^{1}$, W. Schütte ${ }^{1}$ (Sektion Pneumologische \\ Onkologie), S. Diederich², J. Biederer'2, C.-P. Heussel², H.-U. Kauczor², D. Wormanns², B. Rehbock², R. Loose² \\ Für die Deutsche Gesellschaft für Pneumologie und Beatmungsforschung \\ Für die Deutsche Röntgengesellschaft, Arbeitsgemeinschaft Thoraxdiagnostik
}

Bibliografie

DOI http://dx.doi.org/

10.1055/s-0030-1256112

Pneumologie 2011; 65:

5-6 () Georg Thieme

Verlag KG Stuttgart · New York

ISSN 0934-8387

\section{Korrespondenzadresse}

Priv.-Doz. Dr. Martin Reck

Oberarzt

Leitung Studiensekretariat

Onkologie

Onkologischer Schwerpunkt

Krankenhaus Großhansdorf

Wöhrendamm 80

22927 Großhansdorf

dr.martin.reck@web.de
Die Deutsche Gesellschaft für Pneumologie und Beatmungsmedizin und die Deutsche Röntgengesellschaft haben in der Vergangenheit nicht empfohlen, radiologische Untersuchungen (Röntgenthorax, CT Thorax) zur Früherkennung von Lungenkarzinomen bei asymptomatischen Personen einzusetzen. Es sollten vielmehr die Ergebnisse prospektiver, randomisierter multizentrischer Studien abgewartet werden, die geprüft haben, ob durch ein Screening eine Reduktion der Mortalität am Lungenkarzinom erreichbar ist.

Die größte derartige Studie, der National Lung Screening Trial des National Cancer Institute der USA, hat nun vorläufige Ergebnisse mitgeteilt, die zeigen, dass jährliche Niedrigdosis-CT's bei starken aktiven oder ehemaligen Rauchern zwischen 55 und 74 Lebensjahren mit einem Nikotinkonsum von mindestens 30 Packungsjahren (z.B. je eine Packung Zigaretten pro Tag 30 Jahre lang oder 3 Packungen pro Tag 10 Jahre lang) zu einer Reduktion der Mortalität am Lungenkarzinom um ca. 20\% gegenüber einem Vergleichskollektiv führen, das regelmäßig mit Thoraxübersichtsaufnahmen untersucht wurde (http://www.cancer.gov/ newscenter/pressreleases/NLSTresultsRelease).

Eine ausführliche Publikation der Ergebnisse ist für 2011 angekündigt.

Vor diesem Hintergrund werden einerseits Anfragen von starken Rauchern selbst oder auch Überweisungen an Radiologen zur Durchführung entsprechender Niedrigdosis-CT's zunehmen.

Da die unkontrollierte Anwendung der Methode außerhalb der exakt standardisierten Studienabläufe jedoch mehr Schaden als Nutzen anrichten kann, haben die AG Thoraxdiagnostik der Deutschen Röntgengesellschaft und die Sektion Pneumologische Onkologie der Deutschen Gesellschaft für Pneumologie und Beatmungsmedizin die folgende vorläufige Empfehlung entwickelt. Diese wird bei Vorliegen weiterer Daten aktualisiert werden.
Empfehlung (Stand 16. 12. 2010)

$\nabla$

Eine native Niedrigdosis-CT des Thorax kann im Einzelfall (im Sinne einer individuellen Früherkennungsuntersuchung) gerechtfertigt werden, wenn folgende Standards einer Qualitätssicherung berücksichtigt werden:

1. Ausführliches Aufklärungsgespräch mit der betreffenden Person

a) Dieses muss Alter und Ausmaß des Nikotinabusus erfassen, es sollte dargestellt werden, dass ein potentieller Nutzen der Untersuchung nur für die Altersgruppe von 55 bis 75 Jahren mit mindestens 30 Packungsjahren nachgewiesen wurde.

b) Es ist ausdrücklich darauf hinzuweisen, dass die sicherste Methode, den Tod am Lungenkarzinom zu vermeiden, darin besteht, das Rauchen aufzugeben gegebenenfalls mit Hilfe entsprechender Rauchentwöhnungsprogramme.

c) Notwendig ist eine ausführliche Aufklärung über die Besonderheiten von Früherkennungsuntersuchungen bei asymptomatischen Personen gegenüber diagnostischen Verfahren bei symptomatischen Patienten mit Nennung folgender Punkte:

- hohe Wahrscheinlichkeit von Kontrolluntersuchungen wegen gefundener Auffälligkeiten

- unnötige Beunruhigung

- Eingriffe und Operationen aufgrund falsch-positiver Befunde

- falsches Sicherheitsgefühl und Verzögerung der Diagnose durch falsch-negative Befunde

- Verlust an Lebensqualität durch lediglich Vorverlegung der Diagnose, bei denjenigen Patienten, die trotz Früherkennung nicht geheilt werden können

- Strahlenexposition wiederholter Niedrigdosis-CT's mit der Möglichkeit von Krebsinduktion 
Diese Aufklärung muss dokumentiert werden. (siehe auch die ausführliche Beschreibung und Diskussion der Inhalte im Internet - http://www.drg.de/ag/thorax/docs/aktuelles/2010/ aufklrungsbogen\%20niedrigdosis-ct\%20der\%20ag\%20thorax diagnostikweb.pdf)

2. Einhaltung einer strikten Qualitätssicherung für den gesamten Prozess von der Aufklärung über Untersuchung, Befundung bis zur Empfehlung weiterführender Maßnahmen:

a) Standardisierte Untersuchungstechnik entsprechend den Empfehlungen der AG Thorax (http://www.drg.de/ag/thorax/docs/aktuelles/2010/ctprotokolle\%20fr\%20screeningweb.pdf)

b) Standardisierte Auswertung mit klaren diagnostischen Algorithmen (Kriterien für unauffälligen Befund, Befunde, die eine Verlaufskontrolle erfordern und Befunden, die einer unmittelbaren Abklärung bedürfen) gemäß den Leitlinien der Fleischner Society, des American College of chest physicians oder der deutschen S3-Leitlinie (https://www.thieme-connect.de/ejournals/pdf/10.1055/ s-0029-1243837.pdf),

http://www.uni-duesseldorf.de/AWMF/11/020-007l.pdf, http://chestjournal.chestpubs.org/content/132/3_suppl/ 108S.long,

http://radiology.rsna.org/content/237/2/395.full.pdf+html, c) Obligat interdisziplinäre Entscheidung zu weiterführenden Maßnahmen in einer Abstimmung zwischen Radiologen, Pneumologen, Thoraxchirurgen und ggf. Pathologen.

d) Standardisierte Dokumentation von Aufklärung, Untersuchungstechnik, einschließlich Archivierung aller Ausgangsschichtaufnahmen, Auswertung und ihrer Ergebnisse sowie Empfehlungen zum weiteren Vorgehen.

e) EDV-gestützte Erfassung der Daten unter 1.-3. in einer Datenbank oder einem Register.

Eine regelmäßige Aktualisierung der Inhalte (spätestens im Frühjahr 2011 nach Veröffentlichung weiterer Ergebnisse des NLST und anderer relevanter Studien) ist geplant und wird im Internet in jeweils aktuellster Fassung verfügbar sein (http://www.drg.de/ ag/thorax/component/content/article/8/279). 